\title{
EDUCAÇÃO A DISTÂNCIA NO PANORAMA DA ENGENHARIA CIVIL: UMA PROPOSTA PARA PROFESSORES E TUTORES TRABALHAREM COM FERRAMENTAS INTERATIVAS.
}

\author{
SÃO PAULO/SP JULHO/2018 \\ Rosilene Aparecida Sousa - UNICSUL - rosilene.vaqueiro@gmail.com \\ Cristiane Camilo Hernandez - UNICSUL - cristiane.camilo@cruzeirodosul.edu.br \\ Tipo: Investigação Científica (IC) \\ Natureza: Relatório Final de Pesquisa \\ Categoria: Pesquisa e Avaliação \\ Setor Educacional: EDUCAÇÃO SUPERIOR
}

RESUMO

\begin{abstract}
O curso na área de Engenharia civil no ensino a distância, vem sendo discutido pelos Conselhos Regionais de Engenharia e Agronomia, por isso a importância de tratarmos o assunto de forma eficaz. $O$ artigo foi elaborado no intuito de auxiliar, por meio de programas específicos de cada área, 0 discente/tutor nas disciplinas de exatas e específicas da área de Engenharia Civil, no Ensino a Distância, foi analisado uma forma coerente de realizar a mediação da disciplina junto ao aluno, utilizando a tecnologia para ampliar o conhecimento na área técnica, motivando-os no conhecimento de programas da área estudada, participando na avaliação da aprendizagem e principalmente sendo capaz de orientar e proporcionar ao aluno condições de uma aprendizagem autônoma, utilizando as mídias interativas, realizando então uma tutoria ativa.
\end{abstract}

Palavras-chave: Engenharia Civil. Educação à distância. Tecnologia. 


\section{INTRODUÇÃO}

O Ensino a distância (EAD) tem como objetivo promover uma formação acessível e de qualidade, proporcionar a educação formal aos cidadãos de qualquer localidade. Um dos grandes desafios é propiciar conteúdo em um ambiente de diálogo, na plataforma virtual. O aluno que busca a graduação na área de Engenharia tem consciência que o estudo é focado na área técnica, sendo assim, a busca por conhecimento e metodologias surge incessantemente, conforme as novas tecnologias aparecem, esperase que o aluno se aperfeiçoe e exercite o conteúdo apresentado na disciplina.

É importante salientar, para aqueles que procuram a área de Engenharia Civil, ao finalizar o curso, serão fiscalizados pelo CREA (Conselho Regional de Engenharia e Agronomia).

Com o intuito de aperfeiçoar a utilização do AVA (Ambiente Virtual de Aprendizagem), deve-se fazer o uso das TIC's (Tecnologias de Informação e Comunicação), pois esse recurso amplia o método de ensino-aprendizagem, tornando a metodologia de ensino dinâmica e flexível e promovendo conhecimento. Segundo Mota (2016),

\footnotetext{
"a formação de cidadãos aptos a desempenhar tarefas complexas e dispostos a enfrentar os desafios das novas e desconhecidas demandas, por meio do uso intenso e consciente de tecnologias inovadoras, é essencial para a educação contemporânea".
}

Isto posto, o presente estudo tem por objetivo apresentar uma proposta para professores e tutores fazerem uso de recursos interativos disponíveis nos ambientes virtuais de aprendizagem (AVA).

\section{EDUCAÇÃO A DISTÂNCIA NO PANORÂMA DA ENGENHARIA}

Conforme Filho (2011), o registro profissional, no caso de cursos superiores e cursos técnicos da área de engenharia, é realizado no Concelho Regional de Engenharia, Arquitetura e Agronomia (CREA), conferindo ao profissional a habilitação necessária, especificando as áreas e os limites de suas atribuições profissionais.

Por isso, a importância do reconhecimento das instituições de ensino e dos cursos de educação a distância (EAD) pelos órgãos competentes (Ministério da Educação, Secretarias de Educação, Conselho Nacional de Educação, etc). As Instituição de Ensino Superior (IES) são fiscalizadas conforme a Lei de Diretrizes e Bases da Educação (LDB), no 9.394, de 20 de dezembro de 1996, e passou pela última atualização pelo Decreto nº 5.622, de 19 de dezembro de 2005. 


\section{METODOLOGIA}

Para a realização desse artigo, foi analisada a vivência dos alunos de engenharia civil, no ensino online, na plataforma Blackboard e o plano de ensino do Curso EAD da Universidade Cruzeiro do Sul Educacional. Verificou-se a postura dos alunos, diante das dificuldades nas disciplinas específicas do curso. Foram pesquisados recursos tecnológicos, como o objetivo de mediar o aluno utilizando essas inovações técnicas no entendimento do conteúdo apresentado no material didático, complementando o conhecimento, por meio das TIC's.

Todas as ferramentas (aplicativos) foram analisados por meio da leitura dos manuais disponíveis nos sites e livros técnicos da Biblioteca Virtual da Cruzeiro do Sul Educacional. O intuito de utilizar essas ferramentas são aproveitá-las na aplicação de exercícios no AVA, muitas dessas ferramentas já existem na plataforma de ensino, porém precisam ser exploradas. Referente ao conhecimento das disciplinas especifica de engenharia civil a utilização de softwares da construção civil, deve ser agregada, por meio de convênios com os administradores desses programas, pois os valores para aquisição são altos, no entanto existem alguns softwares que possuem a versão estudante e outros são gratuitos.

\section{EDUCAÇÃO A DISTÂNCIA COM O AVANÇO DA TECNOLOGIA}

Segundo Omura (2016. p.87), com a expansão das tecnologias de informação e comunicação pela sociedade, as formas de ensinar e aprender se modificaram. Tanto professores como alunos têm contato frequente e diário com as mais diferentes mídias.

Com todo esse avanço da tecnologia, as IES vem trabalhando para mostrar excelência no ensino $E A D$, pois o mesmo vem se popularizando, conforme entrevista do professor Dr. Márcio Luiz de Andrade Netto (Netto, 2015) ao site Apeoesp (Associação profissional dos engenheiros agrimensores no estado de São Paulo), ele fez a seguinte citação neste contexto:

\footnotetext{
"Sem a educação à distância a gente não vai progredir na área educacional. Instalar universidade é caro. O ensino superior presencial de boa qualidade vai ficar restrito às universidades já existentes e que são consideradas boas já há algum tempo. As novas vagas que têm que surgir, especialmente na área de engenharia, virão com certeza através do ensino à distância, que é uma maneira de você ter qualidade e alcance geográfico e social. Estas são as premissas em que a gente se baseia. As críticas vêm principalmente por desconhecimento. As metodologias de ensino garantem qualidade ao curso dado na forma EAD $90 \%$ do mercado ainda não usa esse tipo de metodologia, poucas instituições fazem isso. Mas a EAD já está se popularizando, principalmente na área de ensino de pós-graduação, em amplo sentido, e educação continuada, isso já vem sendo feito com muita competência por muitas instituições daqui. O que a gente quer garantir agora é qualidade na graduação, esse é 0 nosso objetivo principal. Tirar de vez a má vontade que existe naturalmente na sociedade".
} 
A qualidade que se espera do EAD nas áreas de engenharia deve ser dada pela metodologia de ensino da instituição, ou seja, um esquema produtivo do material didático elaborado por com professores especialistas na área, plataforma de ensino dinâmica e tutores com currículo profissional da área. A universidade deve ter o objetivo de promover o conhecimento e avaliar se o aluno está adquirindo aprendizado com excelência.

\section{PERFIL DO ALUNO DE EXATAS NO EAD}

O sucesso do aluno no EAD depende de seu comprometimento, pois ele tem que se organizar nas rotinas de estudo, ter um planejamento do tempo se dedicando a leitura do material didático, materiais complementares, atividades, entre outros, no entanto com liberdade em seus horários de estudos

Os alunos de exatas demonstram um perfil muito dinâmico, sendo assim a forma como interagir com eles também deve ser desta forma, as apostilas devem conter além de todo embasamento teórico, exercícios de fixações, com exemplos de resolução, além de caminhos alternativos para que eles possam resolver exercícios utilizando a tecnologia. Exemplos, materiais complementares como softwares específicos, tutorias para calculadoras e programas. Como forma de incentivar a utilização desses equipamentos no seu cotidiano, as apostilas deverão conter a resolução de exercícios, exemplificando com a utilização das ferramentas.

O incentivo na utilização de softwares da área técnica também deve ser feito por meio de cursos livres e indicação do professor tutor para cada área estudada, a citação abaixo, complementa esse capítulo com a seguinte citação:

\footnotetext{
Nesse contexto de mudança paradigmática, as universidades, seus gestores e seus professores precisam refletir sobre as reais necessidades que os alunos irão enfrentar em suas profissões e em suas vidas. A sociedade do conhecimento vem trazendo novos enfrentamentos para a população, pois as exigências na formação de cada área profissional tendem a mudar, e o aluno precisa estar preparado para essas transformações. Portanto, a formação deve contemplar um espaço aberto para o diálogo, para a busca incessante do novo, do desejo de pesquisar e tornar-se autônomo e produtivo, BRASIL (1996).
}

\section{UTILIZAÇÃO DAS TIC’S NO ENSINO A DISTÂNICA}

$\mathrm{Na}$ área de exatas, a utilização da interatividade com demonstrações matemáticas e atividades estruturadas deverão ser praticadas com fins didáticos e com o objetivo focado no desenvolvimento dos alunos. Essa interatividade deve atrai-los pelo conhecimento, utilizando a tecnologia a favor do aprendizado e capacitando-os para o mercado de trabalho. A seguir apresentamos ferramentas tecnólogicas (Quadro 1) que 
auxiliam o professor/tutor a ensinar o conteúdo da matéria.

\section{Quadro 1. Ferramentas tecnológicas:}

\begin{tabular}{|c|c|c|}
\hline APLICATIVOS & OBJETIVO & TUTORIA ATIVA \\
\hline $\begin{array}{c}\text { EXPLAIN } \\
\text { EVEYTHING }\end{array}$ & $\begin{array}{c}\text { Criar e gerenciar projetos, interface para } \\
\text { trabalhar com monitor touchscreen. }\end{array}$ & $\begin{array}{c}\text { Escrever ou desenhar da mesma } \\
\text { forma que o faria em uma folha de } \\
\text { papel. }\end{array}$ \\
\hline SMART BOARD & $\begin{array}{c}\text { Quadro interativo interface para trabalhar } \\
\text { com monitor touchscreen. }\end{array}$ & $\begin{array}{c}\text { Apresentar o material, utilizando } \\
\text { imagens onde os alunos poderão } \\
\text { interagir fisicamente com as aulas, } \\
\text { movendo palavras, números e } \\
\text { imagens. }\end{array}$ \\
\hline $\begin{array}{c}\text { KALTURA E } \\
\text { RECORDEN }\end{array}$ & $\begin{array}{c}\text { Repositório de vídeo, ele tem interface } \\
\text { com várias ferramentas } e \text {-learning, dentro } \\
\text { do Blackboard. }\end{array}$ & $\begin{array}{c}\text { Criar e armazenar vídeos e com o } \\
\text { auxílio da ferramenta screen } \\
\text { recording. }\end{array}$ \\
\hline MATHTYPE & $\begin{array}{c}\text { Editor de equaços matemáticas, } \\
\text { permitindo criar equações complexas. }\end{array}$ & $\begin{array}{c}\text { Facilitar a criação do material } \\
\text { didático na área de exatas. }\end{array}$ \\
\hline $\begin{array}{c}\text { DAUM EQUATION } \\
\text { EDITOR }\end{array}$ & $\begin{array}{c}\text { Possibilita a escrita rápida de equações, } \\
\text { podendo salvar como texto ou imagens } \\
\text { para utilizar posteriormente nos } \\
\text { documentos. }\end{array}$ & $\begin{array}{c}\text { Permitir criação de trabalhos } \\
\text { como, projetos e artigos científicos } \\
\text { na área de exatas pelos alunos }\end{array}$ \\
\hline $\begin{array}{c}\text { REALIDADE } \\
\text { AUMENTADA }\end{array}$ & $\begin{array}{c}\text { Baseado em imagens gráficas 3d geradas } \\
\text { por computador em tempo real. }\end{array}$ & $\begin{array}{c}\text { Auxiliar os alunos na percepção, } \\
\text { apreciação, aperfeiçoando a } \\
\text { visualização de formas } \\
\text { arquitetônicas. }\end{array}$ \\
\hline
\end{tabular}

Conforme Corrêa (2001), um Laboratório Virtual de Aprendizagem (LVA) tem como objetivo principal complementar a construção do conhecimento do estudante, por meio do reconhecimento explícito dos processos envolvidos na atividade de ensino. Apresentamos no Quadro 2 alguns LVA's e suas funcionalidades nas disciplinas de Física, Química, Química Orgânica, Física e Biologia.

\section{Quadro 2. Laboratórios Virtuais de Aprendizagem}

\begin{tabular}{|c|c|c|}
\hline LABORATÓRIO & DESCRIÇÃO & URL \\
\hline VIRTUALLAB & $\begin{array}{c}\text { Simulações realistas que } \\
\text { abrange os principais recursos } \\
\text { de um laboratório. Os usuários } \\
\text { são expostos a um ambiente } \\
\text { virtual no qual podem fazer } \\
\text { escolhas e tomar decisões. }\end{array}$ & http://virtuallab.pearson.com.br/ \\
\hline Projeto UAITEC & $\begin{array}{c}\text { Materiais didáticos interativos: } \\
\text { simulações e jogos. }\end{array}$ & http://www.uaiteclab.mg.gov.br/inicio/uaitec \\
\hline CHEMCOLLECTIVE & $\begin{array}{c}\text { Projetado para cálculos } \\
\text { químicos, manipulação de } \\
\text { maneira semelhante a um } \\
\text { laboratório real. }\end{array}$ & http://www.chemcollective.org/ \\
\hline
\end{tabular}

Segundo Amaral, et al (2011), os LVA's consistem em plataformas digitais oferecidas com o intuito de dar suporte à realização de experiências sem a necessidade da 
presença do usuário em um determinado local, tal como ocorre no contexto dos laboratórios reais.

\section{SOFTWARES DA ÁREA DE ENGENHARIA CIVIL}

Segundo Onishi (2017), como progresso de todas as áreas de atividades profissionais, as técnicas não podiam mais progredir apenas com as regras empíricas. As regras de trabalho passaram a ser apoiadas e justificadas pelos conhecimentos científicos. As técnicas ganharam, então, o nome de tecnologias. Como o objetivo é instigar os alunos a conhecerem as tecnologias aplicadas na Engenharia Civil, a universidade pode desenvolver cursos livres, como forma de aplicar uma pequena introdução sobre essas ferramentas. No entanto vale ressaltar a frase abaixo do Engenheiro estrutural Nelson Covas (Covas, 2016):

\footnotetext{
Software não elabora projetos. O software e apenas uma ferramenta para cálculo e geração de desenhos de elementos estruturais. Esses desenhos somente se tornam desenhos representativos de projeto após a análise, verificação e validação da sua exatidão. Os softwares ainda não estão no estágio de equacionar adequadamente todas as variáveis presentes num projeto. O computador e o software não são substitutos para o conhecimento, a experiência e a criatividade do engenheiro.
}

As disciplinas específicas de engenharia civil, possuem muitos softwares que ajudam no cálculo, planejamento e concepção de um projeto, sendo assim, o incentivo na utilização desses softwares em atividades complementares, por meio da aplicação de cursos livres a distância, irão introduzir os alunos no mercado de trabalho. No Quadro 3 apresentamos uma relação de programas para as disciplinas de Engenharia Civil:

\section{Quadro 3. Disciplinas e programas}




\begin{tabular}{|c|c|c|}
\hline DISCIPLINA & PROGRAMA & URL \\
\hline \multirow{3}{*}{ Análise Estrutural } & Ftoll & https://webserver2.tecgraf.puc-rio.br/ftool/ \\
\hline & SAP 2000 & https://www.csiamerica.com/products/sap2000 \\
\hline & Ansys & https://www.ansys.com/products/structures \\
\hline \multirow{3}{*}{ Cálculo Estrutural } & TQS & $\begin{array}{l}\text { http://www.tqs.com.br/index.php/produtos-e-solucoes } \\
\text { /sistemas-em-conjunto/cadtqs-estudante }\end{array}$ \\
\hline & Cypecad & http://www.multiplus.com/software/cypecad/ \\
\hline & MSCalc & http://www.mscalc.com.br/\#detalhes \\
\hline Projeto de Edificações & AltoQiEberic10 & http://www.altoqi.com.br/eberick-novidades/ \\
\hline $\begin{array}{l}\text { Modulação e } \\
\text { detalhamento }\end{array}$ & QiAlvenaria & $\begin{array}{l}\text { https://www.altoqi.com.br/hotsite/solucao-de-alvenaria- } \\
\text { estrutural/ }\end{array}$ \\
\hline $\begin{array}{l}\text { Edição de armaduras e } \\
\text { detalhamentos }\end{array}$ & QiEditor & https://www.altoqi.com.br/hotsite/qieditor-de-armaduras/ \\
\hline \multirow{4}{*}{$\begin{array}{l}\text { Instalação Elétrica } \\
\text { Predial Baixa Tensão }\end{array}$} & QiElétrico & http://www.altoqi.com.br/qibuilder/\#tab_eletrico \\
\hline & AutoCAD Electrical & $\begin{array}{l}\text { https://www.autodesk.com.br/products/autocad/included- } \\
\text { toolsets/autocad-electrical }\end{array}$ \\
\hline & PRO-Elétrica & $\begin{array}{l}\text { http://www.multiplus.com/software/projetos-eletricos/pro- } \\
\text { eletrica/ }\end{array}$ \\
\hline & CadProj Elétrica & http://www.highlightoficial.com/caddproj-eletrica/ \\
\hline \multirow{4}{*}{$\begin{array}{l}\text { Projeto de instalação } \\
\text { Hidráulicas }\end{array}$} & TigreCad & https://www.tigre.com.br/solucoes/tigre-cad \\
\hline & PRO-Hidráulica & http://www.multiplus.com/software/pro-hidraulica/ \\
\hline & CadProj Hidráulica & http://www.highlightoficial.com/caddproj-hidraulica/ \\
\hline & QiHidrossanitário & https://www.altoqi.com.br/hotsite/qihidrossanitario/ \\
\hline \multirow[t]{2}{*}{ Topografia } & $\begin{array}{l}\text { Bentley } \\
\text { TopoGRAPH }\end{array}$ & http://www.topograph.com.br/ \\
\hline & Posição & http://www.posicao.com.br/ \\
\hline Desenhos 2D & Autocad & https://www.autodesk.com.br \\
\hline $\begin{array}{l}\text { Modelos 3D - } \\
\text { interiores }\end{array}$ & SketchUp & https://www.sketchup.com/pt-BR. \\
\hline \multirow{2}{*}{$\begin{array}{l}\text { Projeto de } \\
\text { Infraestrutura }\end{array}$} & InRoads & $\begin{array}{l}\text { https://www.bentley.com/pt/products/product-line/civil- } \\
\text { design-software/power-inroads. }\end{array}$ \\
\hline & Autocad Civil 3D & $\begin{array}{l}\text { https://www.autodesk.com.br/products/autocad-civil- } \\
\text { 3d/overview: }\end{array}$ \\
\hline \multirow{3}{*}{ Geotécnica } & Adina & http://www.adina.com/industries.shtml\#Civil. \\
\hline & Geo5 & http://geo5.com.br/ \\
\hline & Maccaferri & $\begin{array}{l}\text { https://www.maccaferri.com/br/documentos/design- } \\
\text { software/ }\end{array}$ \\
\hline \multirow{5}{*}{$\begin{array}{l}\text { Planejamento - } \\
\text { Orçamentos }\end{array}$} & MS-Project & $\begin{array}{l}\text { https://products.office.com/pt-br/project/project-and- } \\
\text { portfolio-management-software }\end{array}$ \\
\hline & OrçaFascio & https://www.orcafascio.com/ \\
\hline & GanttProject & http://www.ganttproject.biz/download \\
\hline & Arquimedes & http://www.multiplus.com/software/arquimedes/ \\
\hline & Sienge & https://www.sienge.com.br/materiais-gratuitos/ \\
\hline
\end{tabular}


Nas disciplinas específicas de Engenharia Civil inúmeros softwares podem ser utilizados, desde os que auxiliam no cálculo e planejamento até a concepção de um projeto, sendo assim, o incentivo na utilização desses softwares em atividades complementares, por meio da aplicação de cursos livres a distância, podem introduzir os alunos no mercado de trabalho, ou seja, apresentar aos futuros profissionais as diversas ferramentas que complementam a sua profissão.

\section{CONSIDERAÇÕES FINAIS}

Todas as ferramentas (aplicativos) apresentadas no trabalho, foram analisadas com o intuito de aproveitá-las na aplicação e fixação do conceito da matéria, nos exercícios elaborados no AVA com o objetivo de ampliar o conhecimento do aluno na área técnica de Engenharia Civil.

O artigo visou focar os programas que auxiliam na aplicação das disciplinas de cálculo, física e química, que são matérias básicas para o curso de Engenharia. No entanto notou-se a importância da utilização de softwares da construção civil, levando em consideração as disciplinas específicas do curso de Engenharia Civil, com o intuito de abrir as portas para o futuro profissional, pois a instituição de ensino deve ser atrativa para os estudantes.

\section{REFERÊNCIAS}

Amaral, Érico; Ávila, Bárbara; Zednik, Herik; Tarouco, Liane; Laboratório Virtual de Aprendizagem: Uma Proposta Taxonômica. ARTIGO. RENOTE - Revista Novas Tecnologias na Educação. Dezembro, 2011.

BRASIL. Diretrizes e Bases da Educação Nacional n. 9394/96. Brasília, DF,1996.

Covas, N. Tecnologia da Informação no Projeto e Modelagem de Estruturas de Concreto. In: 58 CONGRESSO BRASILEIRO DO CONCRETO ATIVIDADES TÉCNICAS, CIENTÍFICAS E SOCIAIS DO CONGRESSO DO IBRACON. Ano XLIV 84 OUT-DEZ 2016.

FILHO, Domingos Lima. Projetos de Instalações Elétricas Prediais.12a th, Érica, 06/2011.

MOTA, Ronaldo. Educação a distância no começo estranha se depois entranha-se! 13 de maio/ 2016. Disponível em: http://reitoronline.ig.com.br/index.php/2016/05/13/educac 
ao-a-distancia-no-comeco-estranha-se-depois-entranha-se. Acesso em: 09 mai. 2018.

NETTO, Márcio L. de A. Educação a distância. Crea SP.12 de dez/ 2015.Disponível em:http://homolog.creasp.org.br/noticias/exibe/educacao_a_distancia. Acesso em: 09 mai. 2018.

OMURA, Hélio. Educação a distância: Característica dos cursos de graduação no ensino superior. In: BREDDA, Cristiano A. (Org.) Educação \& Gestão: Modelos Educacionais e métodos de Gestão aplicados à Educação. 1a. ed. Campinas/SP: Lopes, 2017.p.85 - 91.

Onishi, P.B.F, M. Introdução à engenharia de estruturas de concreto. Cengage. 1st edição.Disponível

em:

https://issuu.com/cengagebrasil/docs/introduc_cc_a7a_cc_830_20a_cc_80_20. Acesso em: 09 mai. 2018. 